\title{
Multi-point Interferometric Rayleigh Scattering Using Dual-pass Light Recirculation
}

\author{
Daniel Bivolaru* and Paul M. Danehy ${ }^{\dagger}$. \\ NASA Langley Research Center, Hampton VA, 23681-2199 \\ and \\ Andrew D. Cutler ${ }^{\ddagger}$ \\ *The George Washington University, Newport News, VA 236
}

\begin{abstract}
This paper describes for the first time an interferometric Rayleigh scattering system using dual-pass light recirculation (IRS-LR) capable of simultaneously measuring at multiple points two orthogonal components of flow velocity in combustion flows using single shot laser probing. An additional optical path containing the interferometer input mirror, a quarter-wave plate, a polarization dependent beam combiner, and a high reflectivity mirror partially recirculates the light that is rejected by the interferometer. Temporally- and spatially-resolved acquisitions of Rayleigh spectra in a large-scale combustion-heated supersonic axi-symmetric jet were performed to demonstrate the technique. Recirculating of Rayleigh scattered light increases the number of photons analyzed by the system up to a factor of 1.8 compared with previous configurations. This is equivalent to performing measurements with less laser energy or performing measurements with the previous system in gas flows at higher temperatures.
\end{abstract}

\section{Introduction}

$\mathrm{M}$ olecular-based optical diagnostics techniques capable of obtaining simultaneous measurements of multiple fluid properties are critically important for characterizing hypersonic air-breathing engines. Correlations between those properties lead to a more detailed understanding of complex flow behavior and aid in the development of multi-parameter turbulence models required for supersonic combustion engine flow path predictions. ${ }^{1,2}$

Temporally- and spatially-resolved laser-based techniques are used currently to probe supersonic combustion flows, for example: single-pulse coherent anti-Stokes Raman spectroscopy (CARS) ${ }^{3}$ to measure $\mathrm{H}_{2}$-air supersonic combustion flow temperature and species concentration in a rectangular duct; CARS-IRS ${ }^{4}$ consisting of the combination of an interferometric Rayleigh scattering system (IRS) ${ }^{5}$ with a dual-pump CARS system on a common platform to probe an $\mathrm{H}_{2}$-air axisymmetric supersonic jet flow for temperature, species concentration, and two orthogonal components of velocity; and Rayleigh scattering techniques using a Fabry-Perot interferometer for spectral analysis ${ }^{6}$ to probe $\mathrm{H}_{2}-\mathrm{O}_{2}$ rocket plume flow to determine the average gas temperature, density, and velocity simultaneously.

The Rayleigh scattering technique as described in Reference 5 uses a narrow-band pulsed laser beam to elastically scatter photons from molecules in the measurement volume. Receiving optics collect the Rayleigh scattered light (signal) from multiple angles, mix it every pulse with the probing laser light and analyze it spectrally with a planar interferometer (solid etalon). The spectra is Doppler shifted by a frequency proportional to the bulk velocity, the spectral width is proportional to the square root of the gas temperature, and the number of photons received is proportional to the gas density. To measure velocity, the spectra of the signal are analyzed for the Doppler frequency shift using the probing narrow laser spectrum (recorded simultaneously with the scattered spectrum on each interferogram) as a reference frequency.

One drawback of this interferometric technique is its low collection efficiency and the inefficient spectral analysis of the photons collected. For example for a total of $4 \times 10^{17}$ photons per probing laser pulse deposited at the

\footnotetext{
${ }^{*}$ Research Scientist, The George Washington University/MAE, 17 Langley Boulevard, MS 493, AIAA Member

${ }^{\dagger}$ Research Scientist, NASA LaRC/ASOMB, 17 Langley Boulevard, MS 493, AIAA Associate Fellow.

${ }^{\ddagger}$ Professor, The George Washington University/MAE, 100 Old Oyster Point Road, Suite 200, AIAA Associate Fellow.
} 


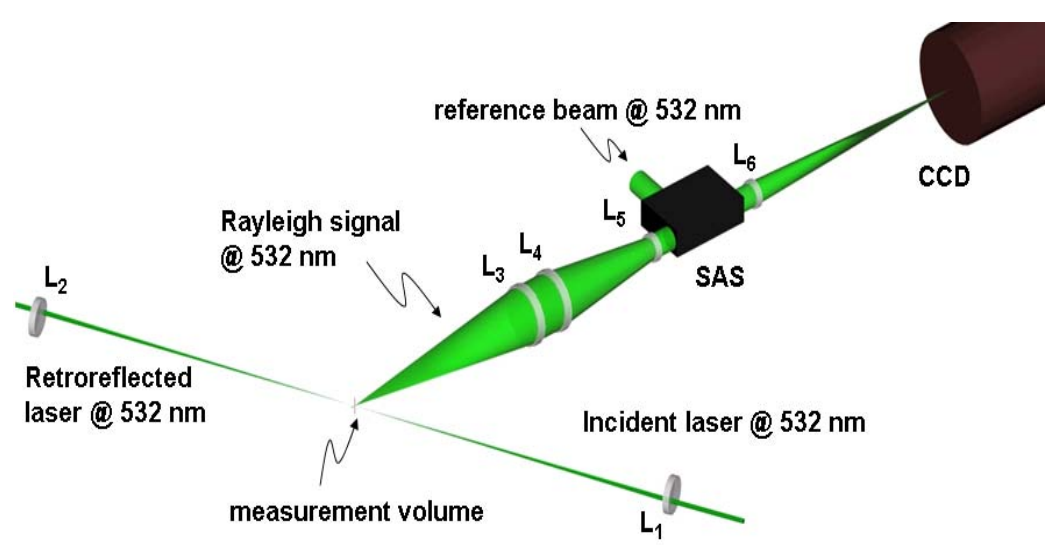

(a)

(b)

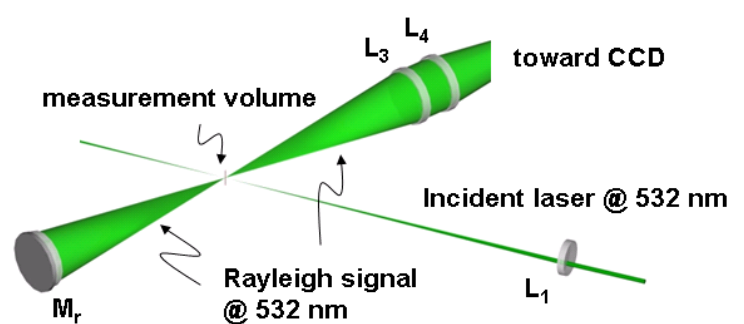

(c)

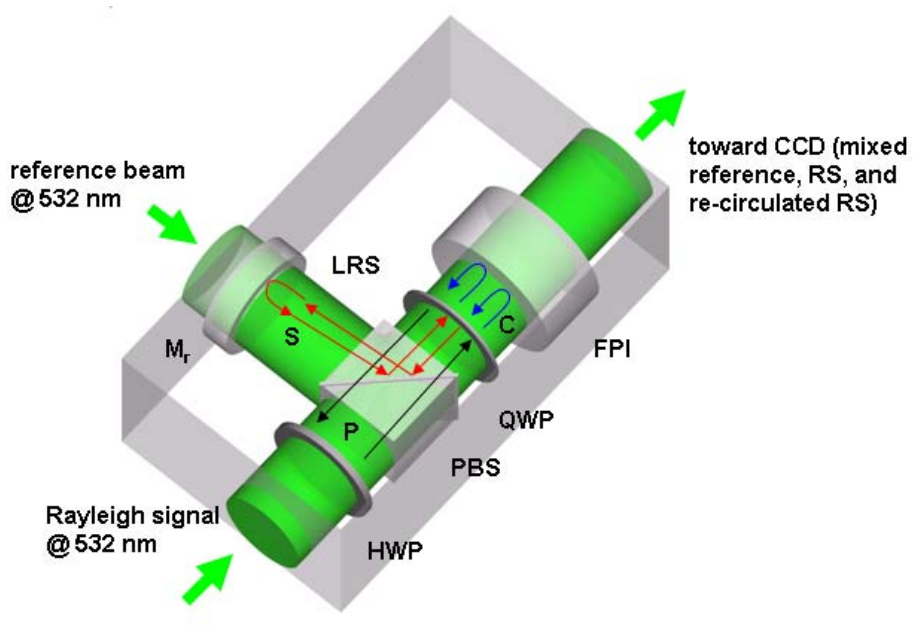

Figure 1. Instrument configurations, (a), using dual laser beams for probing and one collecting optics, (b), using one laser beam for probing and two collecting optics, and (c) the spectral analysis system (SAS) using dual-pass light re-circulation. the instrument to measure at high temperatures without the requirement of increasing the laser energy or knowing the velocity field.

\section{Experimental and Optical Setup}

The technique was developed and the tests were conducted at NASA Langley Research Center's Direct Connect Supersonic Combustor Test Facility (DCSCTF) on an axisymmetric Mach 1.6 $\mathrm{H}_{2}$-air combustion-heated jet flow at sensible enthalpy levels of a Mach 5.5 hypersonic flight. The gas flow is vitiated air with the products of combustion of $\mathrm{H}_{2}$, air and $\mathrm{O}_{2}$ with $23.1 \%$ by mass un-reacted $\mathrm{O}_{2}$. These tests are performed simultaneously with CARS using the combined CARS-IRS instrument that uses a common path green laser beam for probing., ${ }^{4,5}$ (11 orders of magnitude loss!) are normally received by the collecting optics, and only a few thousands are received by the detector. It is known that for constant gas properties and fixed instrument configuration, the number of photons scattered at the measurement volume is directly proportional to the incident laser energy. Obviously, to increase the Rayleigh signal, an increase in the laser energy deposited at the measurement location is required, and this method is mostly implemented.

Unfortunately, laser induced gas breakdown limits the laser beam energy deposited at the measurement volume. One solution is to use an energy storage system or a pulse stretcher to deliver additional energy in bursts of pulses delayed in time to avoid the gas breakdown. Although this technique is relatively simple to implement, it is challenging when used in harsh environments, such as supersonic combustion test facilities, mostly due to the influence of acoustic noise and vibrations. Another way to increase signal is to recover the signal that is rejected by the spectral analysis system (required to analyze the Rayleigh signal) which usually contains a high reflectivity planar interferometer. In certain cases of predictable velocity fields this challenging problem can be amended by using a secondary cavity to reflect the rejected light back to the spectral analysis system. ${ }^{7}$

Here, we present a novel configuration of the IRS system using a planar etalon and a dual-pass light recirculation system (IRS-LR) used to increase the signal intensity thereby the accuracy and the applicability of

measurement volume, only few million 


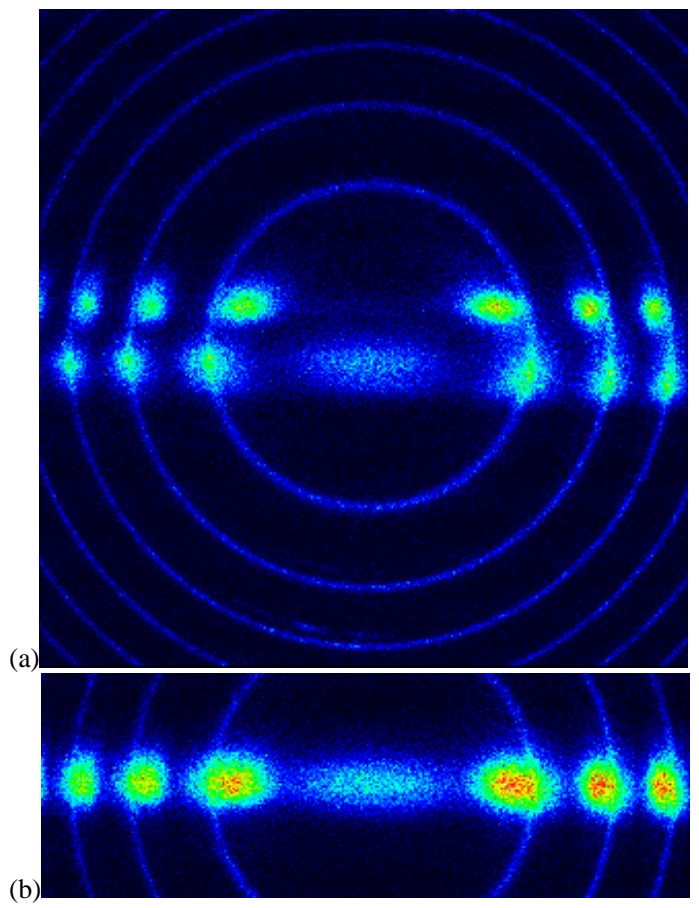

Figure 2. Single-shot interferograms containing reference laser frequency (concentric ring pattern) and Rayleigh scattering spectral information (horizontal pattern) used to solve directly for two orthogonal components of velocity. (a), spatially separated spectra, and (b) overlapped spectra.

molecules, is collected by a the This collected scattered light is then collimated to a smaller diameter by lenses $\mathrm{L}_{4}$ and $\mathrm{L}_{5}$, combined with a fraction of the main laser light using a beam combiner, and passed through a solid etalon for spectral analysis. The last two optical components are part of the spectral analysis system (SAS) hardware. The interference fringe pattern formed by the lens $\mathrm{L}_{6}$ at the output of the etalon is recorded by a CCD camera with electron multiplication gain. Synchronization pulses are used for the camera to match the laser pulse repetition rate of $20 \mathrm{~Hz}$. The resulting interferograms, as shown in Fig. 2 (a), contain spectral information from multiple collecting angles (horizontal patterns) and the laser frequency (concentric ring pattern) used as reference. The figure uses a false color scale. The highest intensity is red followed by yellow, green, blue, and black as the lowest. Each horizontal pattern is the image of the laser beam from one viewing direction (spatially resolved) filtered spectrally by the etalon. The measurement volume resembles the laser beam pattern seen in the interferogram. This pattern defines up to six non-evenly spaced points distributed along $2 \mathrm{~mm}$ around of the laser beam focus. The furthest points in the center of the pattern are situated about $0.8 \mathrm{~mm}$ apart. The bisector of the angle formed by the laser beam and the viewing direction gives the direction of the velocity component being measured. Since the probing laser beams are anti-parallel, the two components of measured velocity are orthogonal. If the incident and retro-reflected laser beams are overlapped at the probe volume the spectra of light scattered from both illumination directions will overlap in the interferogram as shown in Fig. 2(b). If the flow velocity field is unknown, this results in an ambiguity of which velocity component is being measured. Therefore overlapped beams are avoided for this setup.

The second implementation (dual-path collecting optics) is illustrated in Fig. 1 (b). The Rayleigh scattered light is collected from a single laser beam as described earlier but from two opposite directions using the mirror $\mathrm{M}_{\mathrm{r}}$ (concave mirror or the combination of a plane mirror and a lens) for one direction and the lens $\mathrm{L}_{3}$ for the other. Thereafter, both collected beams, directly collected by lens $\mathrm{L}_{3}$ and collected and retroreflected toward lens $\mathrm{L}_{3}$ by the mirror $\mathrm{M}_{\mathrm{r}}$, are combined in a single signal beam, and analyzed spectrally (by the SAS) in the same way as described in the preceding paragraphs. Interferograms that are similar with those shown in Fig. 2(a) are obtained every pulse 
of the laser. As opposed to the first configuration, in this arrangement the signal fringe pattern displays an inward displaced Doppler shifted Rayleigh spectra for a positive velocity component being measured.

If the beams are perfectly overlapped at the measurement volume, by tuning the mirror $\mathrm{M}_{\mathrm{r}}$ such to form a secondary resonant cavity with the input mirror of the interferometer, the arrangement can also re-circulate efficiently the Rayleigh signal light. An increase in intensity of up to 3.5 times was obtained in previous experiments. The interferogram pattern for this setup is similar to the interferogram pattern shown in Fig. 2(b). Practically, the setup can be used to measure two orthogonal velocity components only in certain special cases, e.g. having one component of velocity much bigger in magnitude than the other.

The choice of configuration during experiments depends mostly on the laser beam stability and optical access to the flow from multiple directions. The use of dual-path receiving optics is influenced more by dust particles since the common recorded image apparently contains twice as many particles compared to the dual-path laser probing configuration. On the other hand the dual-path laser probing configuration is more affected by beam spatial instability that is associated with the longer path of the retro-reflected laser beam. This path is usually more than $3 \mathrm{~m}$ longer than the incident laser beam optical path for the beams to be temporally separated (9 nanoseconds pulse width traveling at the speed of light) and to avoid laser induced gas breakdown.

In-house developed image processing software is utilized to extract the scattered light spatial and spectral information for both collecting directions and for the reference laser from each interferogram. These data are fit with simplified Rayleigh scattering models to determine the spectral broadening, the total number of photons, and the Doppler shift frequency of the spectra with respect to the reference laser frequency. The bulk velocity of the flow at multiple locations (defined by the fringe pattern) is calculated from the Doppler shift.

\section{B. Configuration Using Dual-pass Light Recirculation}

In the new implementation of the system using dual-pass light-recirculation sown in Fig. 1 (c), a planar mirror $\mathrm{M}_{\mathrm{r}}$ is situated on a second optical axis perpendicular to the first optical axis and passing through the polarization dependent beam splitter/combiner PBS. A quarter-wave plate QWP, changes the linear polarization of the Rayleigh scattered light beam to circular polarization. In a preferred arrangement ${ }^{8}$, the P-polarized (P) Rayleigh signal that passes through the PBS is changed to circular polarization (C) by the quarter-wave plate QWP. The mirrors of the planar Fabry-Perot interferometer FPI reflect most of the circularly polarized beam energy on the opposite direction on the optical axis toward the QWP. The beam polarization is changed thereafter to S-polarization (S) by the quarter-wave plate QWP, and the beam is reflected by the PBS toward the re-circulating mirror $\mathrm{M}_{\mathrm{r}}$ on a second optical axis perpendicular to the first optical axis. The re-circulating mirror $\mathrm{M}_{\mathrm{r}}$ is adjusted to reflect back the Spolarized beam in opposite direction on the second optical axis toward the PBS. Therefore, the PBS receives for the second time and directs the Rayleigh signal on the initial optical axis toward the interferometer FPI. In the next cycle the beam polarization is rotated back to P-polarization, the beam passes through the PBS on the first optical axis but in opposite direction, and consequently the remaining beam energy is lost. The half wave plate (HWP) adjusts the polarization direction of the input collimated beam for optimum pass at QWP. The high reflectivity mirror $\mathrm{M}_{\mathrm{r}}$ is also used for mixing the reference light with the signal without substantial loss of signal. This is performed by sending a collimated beam on the secondary optical axis through to the back of this mirror. Losses of the reference light by backward reflection caused by this high reflectivity mirror are not important since there is ample unused laser beam intensity available and very little beam energy is required to be transmitted for this purpose.

The dual-pass light re-circulation system can be used in conjunction with any of the previous Rayleigh scattering configurations that uses a planar mirror interferometer or etalon for spectral analysis. The advantage from the practical point of view is its simplicity and robustness. In contrast with the dual-cavity light recirculation, this arrangement is less efficient as it will be shown next.

\section{Results and Discussions}

\section{A. Signal Intensity with and without Light Recirculation}

The peak intensity of the fringe pattern is given by $\quad\left[\mathrm{I}_{\mathrm{T}}\right]_{\max }=\mathrm{I}_{0} \mathrm{~T}^{2} /\left(1-\mathrm{R}^{2}\right)$, where $\mathrm{I}_{0}$ is the input beam intensity, $\mathrm{T}$ is the transmitted, and $\mathrm{R}$ is the reflected intensity, respectively. ${ }^{9}$ In our case for an etalon with a reflectivity of $\mathrm{R}=$ 0.87 , only $6.7 \%$ of the incident light is transmitted. For ideal non-absorbing and non-scattering mirrors the illumination at the peak of the fringes is unchanged by the insertion of the interferometer, but the additional absorption or scatter losses at the surface reduce this intensity by $10-25 \%$ depending on the optical components quality. The reflected intensity is complementary to the transmitted one and is given by $\left[\mathrm{I}_{R}\right]=1-\left[\mathrm{I}_{T}\right]$, which is in our example about $93 \%$ of the incident light. As shown in Fig. 2, the transmitted reference fringe pattern, the Airy 


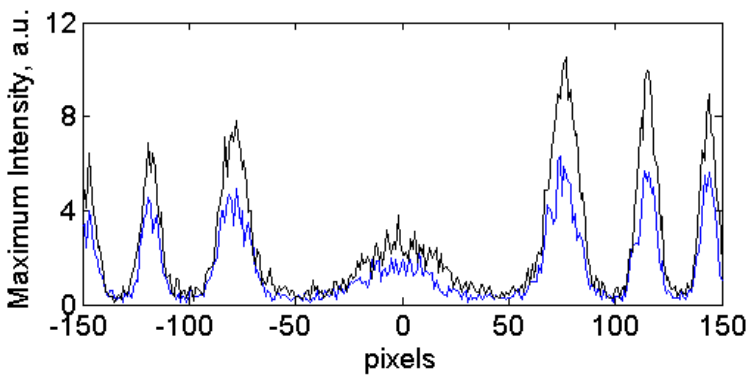

(a)

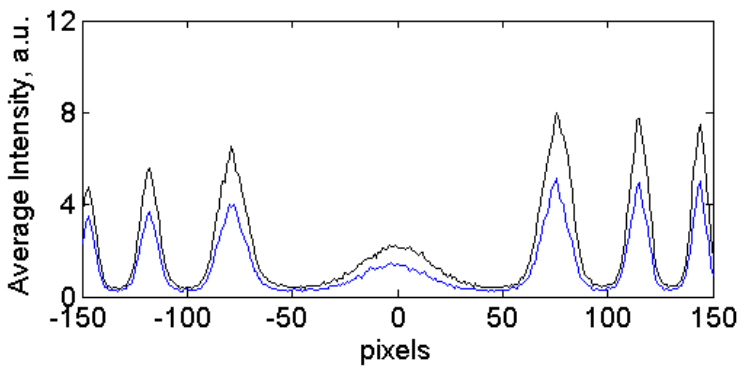

(b)

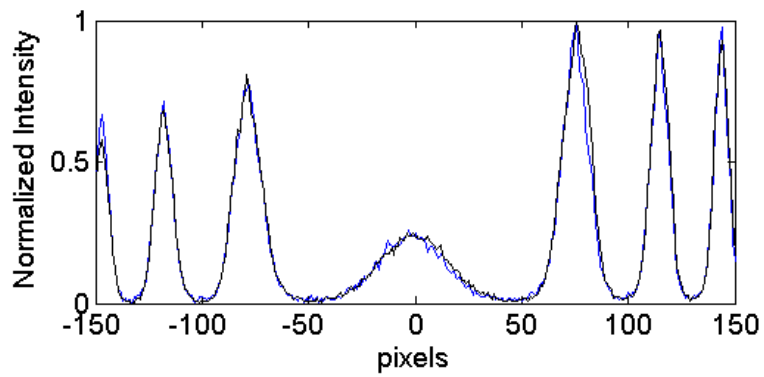

(c)

Figure 3. Single-shot spectra of mixed reference and Rayleigh scattered light with (black) and without (blue) light recirculation. (a) one pixel line, (b) 21 lines average around the row of maximum amplitude, and (c) normalized intensities. Spectra are obtained in stagnant air. instrument function, consists of bright circular pattern against a darker background. In the same way, the reflected pattern is of bright background with narrow dark fringes (much less light) positioned at the same location as the bright fringes of the transmitted pattern.

If the incident beam is perfectly collimated, and if the reflected pattern is retro-reflected for the second time toward the interferometer without loses, the retroreflected beam will have no contribution to the transmitted intensity due to absence of photons in the region of peak intensity in the fringe pattern. Fortunately the degree of collimation is finite and can be modified using lens $\mathrm{L}_{3}$ and also due to unavoidable surface and volume dispersion from optical components the retroreflected beam pattern of sharp dark fringes (usually narrower) is destroyed. Therefore, the retroreflected beam contributes substantially to the increase in intensity of the transmitted fringe pattern.

Indeed, this is demonstrated in Fig. 3 which shows single-shot spectra of Rayleigh scattered light mixed with reference laser light obtained with the second instrument configuration (blue trace) and with the configuration of the instrument that uses the light recirculation technique (black trace). For simplicity, the interferograms were obtained in stagnant air (therefore the spectra have zero Doppler shifts) with the signal pattern (signal spectra) passing through the center of the interferogram. The reference light was reduced to a minimum, mostly to the light level of the background scattered light at the measurement location. The camera offset background was subtracted from each interferogram and the small contribution of the reference light was neglected in the following calculations. Figure 3(a) shows the intensity profile of one pixel row through the maximum intensity region of the signal, and Fig 3(b) shows an average of 21 rows around this row for both cases. The increase in signal intensity (black trace) is evident in these figures. Also it is found that this process has negligible influence on the instrument function. This is demonstrated in Fig. 3(c), which shows the normalized nonlinear spectra of the signal with light recirculation (black trace) and without (blue trace). These two normalized spectra are almost identical.

Table 1 exemplifies the measurements of the maximum $\left(\mathrm{I}_{\mathrm{m}}\right)$ and the total number $\left(\mathrm{I}_{\mathrm{t}}\right)$ of signal photoelectrons generated by the CCD. The regions of interest (ROI) from interferograms that are used for calculations are shown in the insets of the table. If the high reflectivity mirror $M_{r}$ is adjusted such that the recirculated image (RI) of the signal is placed on the interferogram next to the direct image (DI) in two parallel horizontal patterns as shown for a normal configuration in Fig. 2(a), about $71.4 \%$ of the signal is found in the retroreflected image RI.

\begin{tabular}{|c|c|c|c|c|c|}
\hline Interferogram (ROI) & Description & $I_{m}\left(N_{e}\right)$ & $I_{m} /\left.I_{m}\right|_{D I}$ & $\mathrm{I}_{\mathrm{t}}\left(\mathrm{N}_{\mathrm{e}}\right)$ & $I_{t} /\left.I_{t}\right|_{D I}$ \\
\hline & Direct (DI) & 8821 & 1 & $1.35 \mathrm{E}+07$ & 1 \\
\hline & Recirculated (RI) & 6458 & 0.73 & $9.62 \mathrm{E}+06$ & 0.714 \\
\hline & Total Overlapped & 13675 & 1.55 & $2.16 \mathrm{E}+07$ & 1.606 \\
\hline
\end{tabular}

Table 1. The number of photo-electrons received by the CCD camera in a predefined region of interest. 


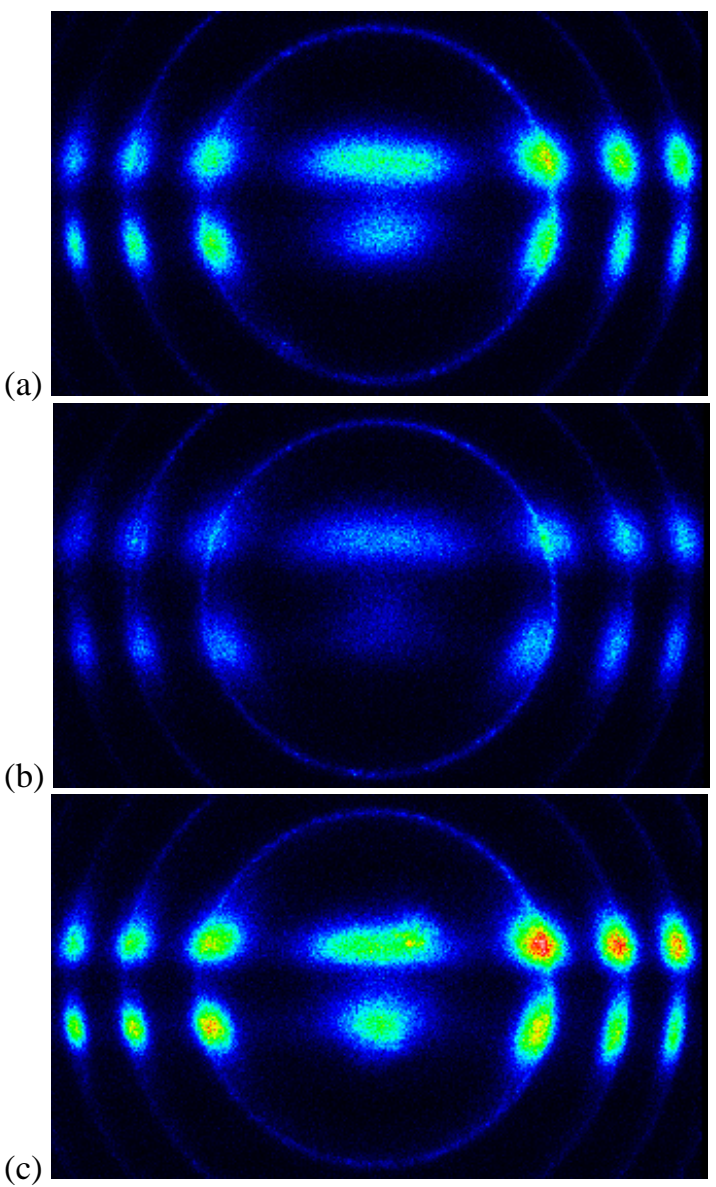

Figure 4. Single shot consecutive interferograms showing changes in flow properties during measurements. (a), $t=t_{0},(b), t=t_{0}+1 / 20$ sec, and (c) $t=t_{0}+2 / 20$ sec. The temporal resolution of each image is about 40 nsec.

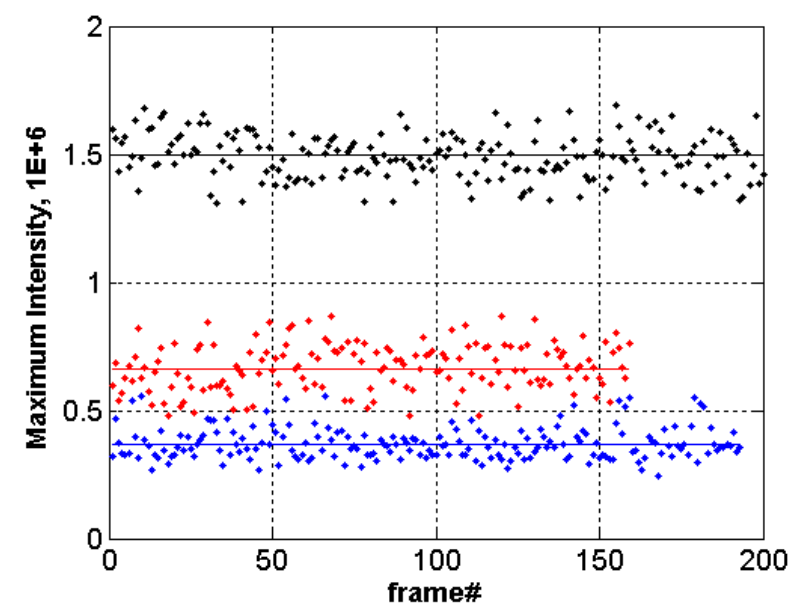

Figure 5. Maximum intensity of Rayleigh scattered light recorded during measurements in a Mach 1.6/Mach 5.5 combustion heated jet compared with cold flow (black symbols). Data are obtained using light recirculation (red), and using the normal configuration (blue).

When these two images, DI and RI, are super imposed on the same interferogram, the combined pattern contains $61 \%$ more photo-electrons than the normal DI image. Although this signal increase is lower than the increase obtained in previous experiments with dual-cavity IRS (of about $85 \%$ ), it is a considerable advantage since it could allow a reduction of the energy of the probing laser by the same amount or an increased range of measurable temperatures using the same laser energy.

Signal intensity measurements are also performed on interferograms obtained in the facility at Mach 1.6/Mach 5.5 enthalpy test conditions. The measurements are performed in the jet's far field at $(\mathrm{x}, \mathrm{r}) / \mathrm{d}=(7.45,-0.35)$, where $\mathrm{x}$ is along the jet axis, $r$ is the radial direction, and $d$ is the nozzle exit diameter $(63.5 \mathrm{~mm})$. The average jet temperature and streamwise velocity are estimated to be $850 \mathrm{~K}$ and $605 \mathrm{~m} / \mathrm{s}$, respectively. ${ }^{10}$ More information about the measured and estimated temperature and velocity values for these experiments can be found in references 5 and11. Three consecutive interferograms containing the signal to solve for two components of flow velocity at up to six points are shown in Fig. 4. The fluctuations in velocity (Doppler shift) and temperature of the gas (spectra broadening) are seen as changes of the signal pattern with respect to the reference pattern. The variations in flow density are seen as variations in signal intensity. As described in the interferogram processing methodology of Reference 5, during the second step, the locations of the laser beams in the interferogram are detected by binning all pixel columns into one averaged column of data. The resulting distribution approximates the intensity distribution across the laser beams. Neglecting the non-uniform background coming from averaging of the reference pattern, the distributions can be fitted with Gaussian functions (the laser beam profile is approximately Gaussian). The locations of the maxima of these distributions give the location of the beams with respect to the center coordinate of the interferogram and are used in the procedure to extract spectra from the interferogram. The integral of these signal functions provide the means of estimating the total number of photons collected by the system. Because the two signals are originating from the same spatial location in the flow, the ratio of these signals (integrals) is approximately a constant independent of the laser energy. This ratio depends only on the optical system and is $1.4( \pm 0.1)$ for the setup using light recirculation and $1.17( \pm 0.1)$ without.

The measurements of intensity for one component of flow velocity are shown in Fig. 5 with light re-circulation (red symbols) and without (blue symbols) together with a measurement using light re-circulation in cold air flow (black symbols) for reference. The averages of these values are shown as straight lines. The cold airflow (subsonic), 
which was used for measurements because it has fewer particles than stagnant air, is obtained by flowing (filtered compressed) air (at room temperature) through the facility nozzle between normal combustion tests. The jet exit velocity is estimated (measured) to be $226 \mathrm{~m} / \mathrm{s}(263 \mathrm{~m} / \mathrm{s})$. The measurement volume for this data is located in the mixing layer at $(\mathrm{x}, \mathrm{r}) / \mathrm{d}=(7.45,-1.24)$, were the flow temperature is about $300 \mathrm{~K}\left(\sim 81^{\circ} \mathrm{F}\right)$ and the magnitudes of velocities are small.

The average maximum intensity of the signal using light re-circulation during this dynamic tests is 1.8 times greater that the average maximum intensity of the signal in the absence of light re-circulation. The intensity variation around the mean represents both the pulse-to-pulse variation of the laser energy and the variation in intensity of the signal due to changes in flow density.

\section{Conclusion}

Dual-pass light recirculation is demonstrated for the first time on an interferometric Rayleigh scattering system. The present configuration provides many advantages, including: instantaneous measurements; simultaneous multipoint measurements along a line; simultaneous measurement of two orthogonal components of velocity; improved stability and accuracy by continuous mixing of the Rayleigh scattered light with the reference laser light; the measurements are not affected by laser frequency drift, interferometer alignment errors, vibrations and background light scattering, and the measurements are not affected by particles scattering at reasonable levels due to direct imaging optics.

Recirculating of Rayleigh scattered light increases the number of photons analyzed by the system up to 1.8 times compared with the previous configurations. This is equivalent to performing measurements with the previous system with less laser energy or in gas flows at higher temperatures.

\section{Acknowledgments}

We wish to thank Diego Capriotti, Tom Mills, and Barry Lawhorne and his team for technical support and operation of the DCSCTF at NASA LaRC. We recognize the important contributions of Joseph Lee, Stephen Jones, James Downey, Markus Weikl, Gaetano Magnotti, Mark Kulick, and Lloyd Wilson during installation, and Sarah Tedder during installation and operation of the combined CARS-IRS instrument in the facility.

This work was supported by the NASA Fundamental Aeronautics Program - Hypersonics Project, Experimental Capabilities and Propulsion Disciplines and through a NASA Research Announcement grant from Experimental Capabilities. Also the project was supported by George Rumford, program manager of the Defense Test Resource Management Center (DTRMC) Test and Evaluation/Science and Technology (T\&E/S\&T) program, under the Hypersonic Test focus area.

\section{References}

1 Drummond, J. P., Danehy, P. M., Bivolaru, D, Gaffney, R. L. Jr., Tedder, S. A., and Cutler, A. D., "Supersonic combustion research at NASA, 2007 Fall Technical Meeting," Eastern States Section of the Combustion Institute, University of Virginia, October 21-24, 2007.

2 Baurle, R.A., "Modeling of High Speed Reacting Flows: Established Practices and Future Challenges,” AIAA 2004-267, 42nd Aerospace Sciences Meeting and Exhibit, Reno, NV, 5-8 Jan, 2004.

3 Cutler, A. D., Danehy, P. M., Springer, R. R., O'Byrne, S., Capriotti, D. P., Deloach, R.,"Coherent Anti-Stokes Raman Spectroscopic Thermometry in a Supersonic Combustor", AIAA Journal, Dec. 2003, Vol. 41, Num. 12, pp. 2451-2459.

4 Bivolaru, D., Lee, J. W., Jones, S. B., Tedder, S., Danehy, P. M., Weikl, M. C., Magnotti, G., and Cutler, A. D., “Mobile Rayleigh - CARS Instrument for Simultaneous Spectroscopic Measurement of Multiple Properties in Gaseous Flows,” $22^{\text {nd }}$ International Congress on Instrumentation in Aerospace Simulations Facilities (ICIASF), Monterey, CA, June, 2007. See also Bivolaru, D, Danehy, P.M., Grinstead, K.D., Jr., Tedder, S., and Cutler, A.D., "Simultaneous CARS and Interferometric Rayleigh Scattering," AIAA-2006-2968, 25 ${ }^{\text {th }}$ AIAA Aerodynamic Measurement Technology and Ground Testing Conference, San Francisco, CA, June 5-8, 2006.

5 Bivolaru, D., Danehy, P. M., Gaffney, Jr. R. L., and Cutler, A. D., "Direct-View Multi-Point Two-Component Interferometric Rayleigh Scattering Velocimeter,” AIAA-2008-0236, 46th Aerospace Sciences Meeting, Reno, NV, January 912, 2008.

6 Seasholtz, R. G., Zupanc, F. J. and Schneider, S. J., "Spectrally Resolved Rayleigh Scattering Diagnostics for HydrogenOxygen Rocket Plume Studies,” J. Propulsion and Power, Vol. 8, No. 5, 1992, pp. 935-942.

7 Bivolaru, D., Danehy, P. M., and Lee, J. W, "Intracavity Rayleigh-Mie Scattering for multipoint, two-component velocity measurement,” Optics Letters, Vol. 31, No. 11, pp. 1645-1647, June, 2006.

8 Bivolaru Daniel, Paul M. Danehy, and Joe W. Lee, NASA Langley Research Center, Hampton, VA, U.S. Patent Application for "Interferometric Rayleigh Scattering Measurement System” Docket No. LAR-17235-1, filed 5 Oct. 2006. 
9 Vaughan, J. M., The Fabry Perot Interferometer, History, Theory, Practice, and Applications, Adam Hilger Series, edited by E.R. Pike and W. T. Welford, Bristol and Philadelphia, 1989, pp. 89-134.

10 Richard, L., Gafney, Jr., "Numerical simulations of a Co-Axial Supersonic-Combusting Free-Jet Experiment," JANNAF2008, Massachusetts, May, 2008.

11 Danehy, P. M., Magnotti, G., Bivolaru, D., Tedder, S., and Cutler, A. D., "Simultaneous Temperature and Velocity Measurements in a Large-Scale, Supersonic, Heated Jet,” JANNAF2008, Massachusetts, May, 2008. 